\title{
HUMAN-AUTOMATION COLLABORATION IN DYNAMIC MISSION PLANNING: A CHALLENGE REQUIRING AN ECOLOGICAL APPROACH
}

\author{
Michael P. Linegang, Heather A. Stoner, Michael J. Patterson \\ Aptima, Inc. \\ Washington, DC \& Woburn, MA
}

\author{
Bobbie D. Seppelt, Joshua D. Hoffman, Zachariah B. Crittendon, John D. Lee \\ University of Iowa \\ Iowa City, IA
}

\begin{abstract}
The US Navy is funding the development of advanced automation systems to plan and execute unmanned vehicles missions, pushing towards a higher level of autonomy for automated planning systems. With effective systems, the human could play a role of mission manager and automation systems could perform mission planning and execution tasks with limited human involvement. Evaluations of the automation systems currently under development are identifying critical conflicts between human operator expectations and automated planning results. This paper presents a model of this human-automation interaction system and summarizes the resulting system design effort. This model provides a theory explaining the source of conflict between human and automation, and predicts that an ecological approach to display design would reduce that conflict. Based on that prediction, the paper describes initial results of an ecological approach to system analysis and design, intended to improve human-automation interaction for these types of advanced automation systems.
\end{abstract}

\section{INTRODUCTION}

Unmanned vehicle systems (UVs) could become "force multipliers" if small teams of human operators could execute complex missions via larger teams of UVs. Parasuraman, Sheridan, and Wickens (2000) describe a scale relating levels of automation (i.e. higher levels of automation = less human involvement required) to four types of information processing (i.e. sensory, perception, decision-making, and response selection). Transforming UVs into true force multipliers requires advanced automation systems with a high level of autonomy for all types of information processing. To support planning and execution of increasingly complex UV missions under the direction of progressively smaller teams of humans, automation must offload information processing burdens from the human. The US Navy is making progress in developing automation to support UV mission planning and execution (e.g., Autonomous Operations Future Naval Capability Intelligent Autonomy [IA] Program). Through IA, technologies are being developed that display "intelligent" behaviors (e.g., optimal task allocation based on humanspecified mission goals, optimal path planning based on knowledge of the environment from net-centric data, real-time coordination of vehicle activities, etc.) and are beginning to offload the human operator in mission planning and execution tasks.

In spite of this progress, lessons learned from IA show that human operator subject matter experts (SMEs) sometimes question mission plans and UV behaviors produced by these automation systems. SMEs have difficulties specifying mission parameters in the manner required by the automation and have difficulties understanding how and why the automation system is generating its plans (Billman, Cristina, Balmer, \& Warner, 2005). These automation systems do not yet support effective human-automation communication and collaboration in dynamic mission planning.

\section{A MODEL OF HUMAN-AUTOMATION INTERACTION}

The key challenge for IA is aligning human conceptualization of the mission planning problem and automation system conceptualization of that problem. Many new automated mission planning and execution technologies use human-specified goals and constraints as the link between humans and automation. Figure 1 is a model of this type of goal-driven mission planning and execution system.

Human operators play two roles in this type of system: 1) specifying goals and constraints for a mission ("Goal Agent”), and 2) reviewing, approving, executing, and/or overriding the automation system's planned actions for achieving those goals, through a variable level of autonomy system ("VLA Agent"). The "Goal Agent" role is the focus for this paper.

The mission planning process begins with the human specifying goals for the automated planning system. The automation system generates a set of planned actions and (assuming no intervention by the "VLA Agent") executes those actions. As those actions are executed in the mission environment, both the human and the automation monitor the environment to identify "error" that would necessitate a modification of the plan.

\section{Conflicting Goals in Human-Automation Interaction}

This model suggests that one source of conflict between human and automation is a result of differences in the error term considered by each agent, and this mismatch is an artifact of the goal specification process. The human and automation both monitor a set of "explicit goals" (e.g. identify threats in a region). But a human commander for a complex mission 


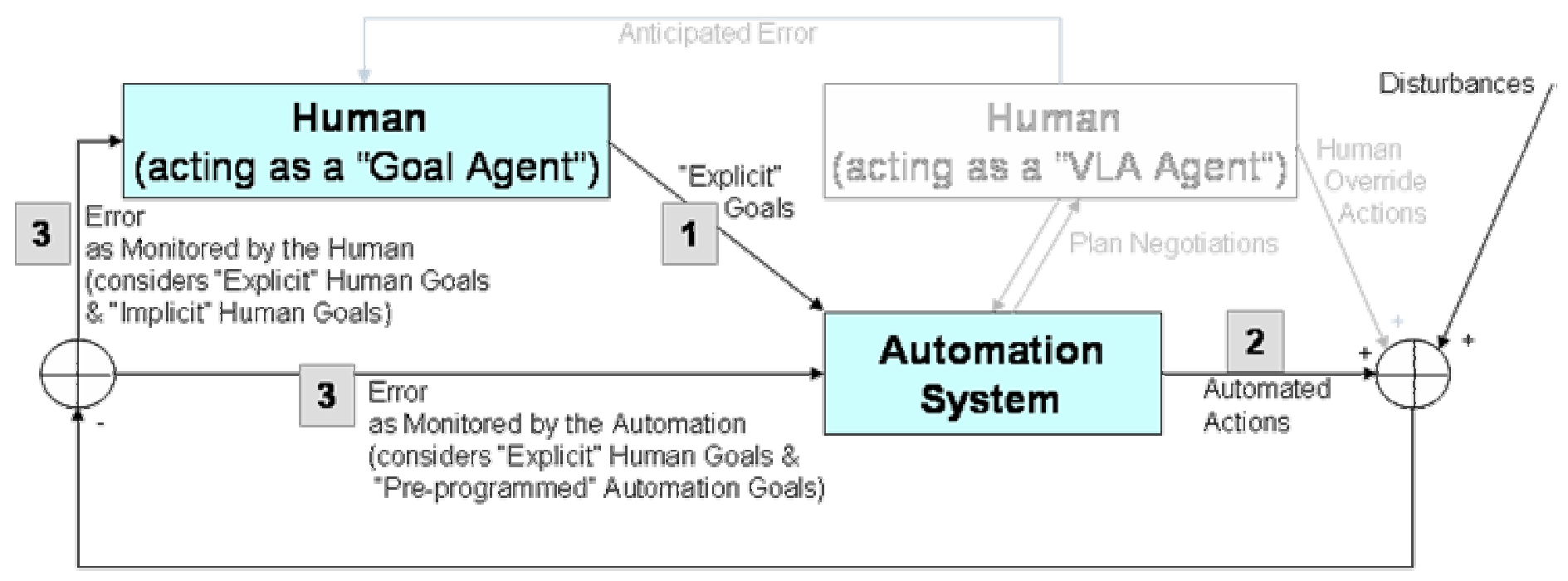

Figure 1 Control theory representation of a goal-driven mixed-initiative system for mission planning and execution

likely also considers other “implicit goals” (e.g. don’t alert an enemy to your presence), and complex automation systems likely also contain "pre-programmed goals" resident in the automated process (e.g. minimize fuel consumption). These "implicit” and "pre-programmed" goals may be shared on any given mission (i.e. for some missions the pre-programmed goals may be very salient to the human as implicit goals for the mission), but in those cases where the human's implicit goals do not match the automation's pre-programmed goals, those differences will result in a different level of "error" seen by the human and the automation. As a result, a mismatch between the operator's awareness of the problem ("explicit” \& "implicit” goals) and the automation system's awareness of the problem ("explicit” \& "pre-programmed” goals) will be amplified as the automation system executes actions targeted to optimize one combined goal set, while the human responds to a different combined goal set.

\section{Addressing Conflicting Goals in Display Design}

This model suggests that a "human-centered" approach to display design is likely to amplify the mismatches between the human and automation. If a mission display is designed to emphasize information that human experts have indicated as being most relevant to the human task, then the display will likely emphasize information relevant primarily to human explicit goals; and perhaps give some emphasis to human implicit goals. While this display may provide excellent support for monitoring aspects of the situation that humans find most relevant, it would likely give even less emphasis to any "pre-programmed goals" that are already of diminished salience for the human. A truly "human-centered" display will not address the problem of mismatches between human and automation conceptualizations of the mission.

To reduce the mismatches between a human operator and an automated planning system, displays are needed that guide human operator attention to features of the problem space that influence automation system functioning in addition to features that are relevant to traditional human approaches. Automated mission planning systems may revolutionize the mission planning process, but revolutionary displays will be required for these systems. If we develop displays that represent information relevant to explicit, implicit, and pre-programmed goals for planning processes, we provide a workspace within which humans and automation can collaborate in optimizing the achievement of all true mission goals (explicit and implicit). An "ecological” approach to design, one that organizes information requirements according to the natural structure of the mission environment, is ideally suited to this problem.

\section{MiDAS: AN ECOLOGICAL APPROACH TO HUMAN- AUTOMATION INTERACTION}

Mission Displays for Autonomous Systems (MiDAS) is a research and development effort designing a collaborationspace for human-automation interaction in mission planning and execution. MiDAS is scoped to a subset of requirements: mission planning for Intelligence, Surveillance, and Reconnaissance (ISR) missions in Littoral regions, where missions are executed by heterogeneous groups of UVs. MiDAS applies an ecological approach; specifically, a comprehensive analysis of the ISR work domain, based on Cognitive Work Analysis (CWA) and Ecological Interface Design (EID) (Vicente, 1999; Burns \& Hajdukiewicz, 2004). This paper describes initial results (a work domain analysis leading to conceptual display designs) and discusses SME feedback.

\section{Analysis of the ISR Work Domain}

Work Domain Analysis (WDA) is a CWA stage that identifies all information categories supporting work in a domain (Vicente, 1999). WDA identifies information types that could potentially influence the processes in the domain (regardless of the level of emphasis human operators give to these information types or processes). WDA organizes these information types into a hierarchical structure. Results from the WDA conducted in the MiDAS effort are shown in Figure 2; an abstraction hierarchy $(\mathrm{AH})$ for ISR mission planning. 


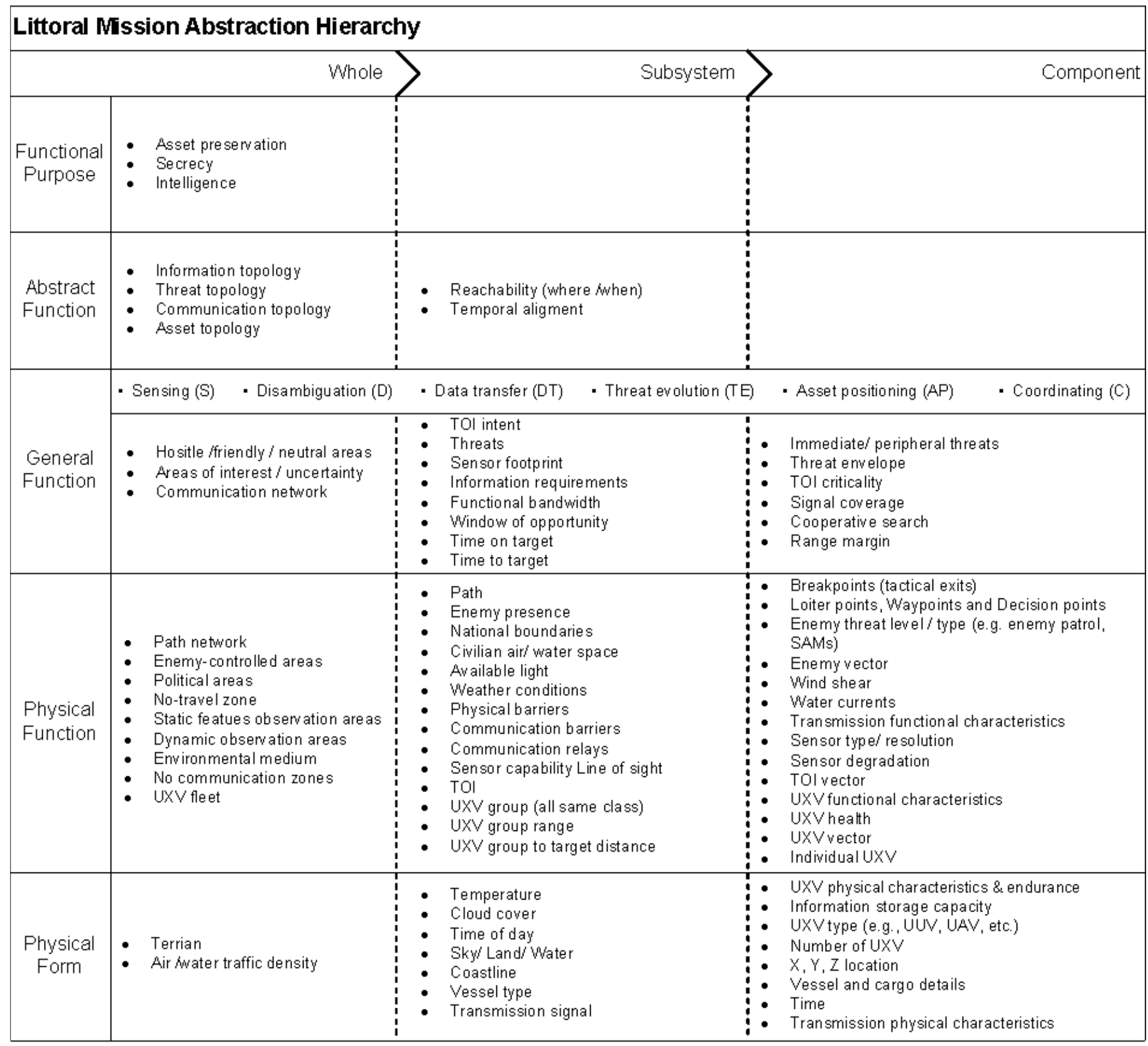

Figure 2. Abstraction hierarchy for littoral ISR missions

Information constructs describing the purpose of ISR missions are represented at the top-left of the AH (i.e. collect Intelligence, while maintaining Secrecy, and Preserving Assets from harm or loss). Progressively more detailed and concrete information constructs supporting those purposeconstructs are mapped to the lower right of the AH (e.g. concrete, physical characteristics of entities in the environment, capabilities and endurance of UV's). The AH provides an organization scheme for information that is salient for human operators, but also structures information that is innate to the processes and transformations that occur in the domain.

The "purpose of the system" constrains system operation. Dynamic systems typically balance two or more competing purposes. We identified three purposes for this system: 1) Intelligence; 2) Asset Preservation; 3) Secrecy. Tersely stated, this system's purpose is to gather intelligence in a manner that preserves assets while maintaining secrecy. The human operator's goals for a mission are likely defined in these terms (e.g. capture pictures of these 5 targets without damaging the UV). The dynamics of this system result from the balancing of competition between intelligence gathering (generally driving assets into harm's way and within detection boundaries) and asset preservation or secrecy (generally pulling assets away from threats and detection boundaries).

\section{Implications for Display Design}

These purposes define the gross level features required for a display designed to support monitoring and control of this system. This system's display needs to convey an 
integrated picture of the intelligence gathering objectives for a mission (likely "explicit" goals of the mission), but these objectives need to be characterized relative to a high level integrated picture of the "threat topology" and "detection boundaries" that impact the secrecy and/or asset preservation functions (likely "implicit” and/or "pre-programmed" goals for the mission). The AH suggests that a properly constructed display will guide the user to an understanding of the problem space at this high level of abstraction, and will facilitate human-automation interaction in a systematic manner relative to these high level properties.

The information in the display should be organized so all information types in the $\mathrm{AH}$ are clustered and organized into the three high level intelligence, threat, and detection features on the display. These features should be decomposable into their component parts, revealing the detailed properties identified in the AH. This type of abstraction-decomposition information architecture would support operators in diagnosing the details of the mission that impact automated planning system functioning and allow the operator to guide automated functioning to relevant information and respond to unexpected events. Since the AH represents all component information constraining the planning process, the comprehensive display based on the AH would support human operator awareness of goals that are influencing the automation system (for better or worse).

\section{DISPLAY CONCEPTS}

Figure 3 and Figure 4 provide an overview of a geographic display concept to support human monitoring of an automated ISR mission planning system. These display concepts were generated based on the above AH. These designs are conceptual, and intended to convey the potential utility of organizing information around the relationships represented in the $\mathrm{AH}$. Many of the detailed design choices (e.g. color palette) do not represent a finished product.

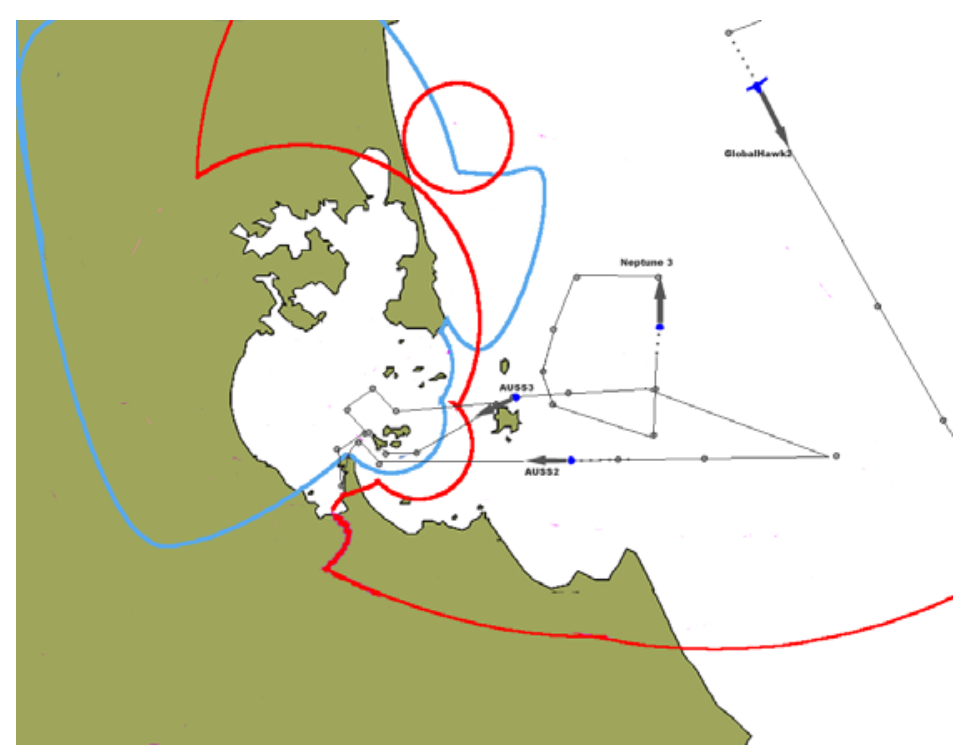

Figure 3. "Secrecy" and "asset preservation" concept

\section{Integrated Presentation of Functional Purposes}

Figure 3 shows a mission environment which emphasizes two of the functional purposes for the ISR mission planning system: 1) maintaining secrecy, and 2) preserving assets. Two different boundaries are displayed $($ Blue $=$ detection boundary for mission secrecy; Red = threat boundary for asset preservation). The blue "secrecy" boundary integrates multiple lower level entities that could impact the secrecy of the mission (e.g., enemy sensor lines-ofsight and sensor footprints relative to available light, weather conditions, physical barriers, etc.). The red "asset preservation" boundary integrates several different types of information: 1) environmental factors that threaten the safety of mission assets (e.g. weather conditions), 2) internal system factors that could threaten the safety of mission assets (e.g. endurance and range margins of UVs, etc.), as well as 3 ) other entities that could threaten the safety of mission assets (e.g. enemy threat envelopes).

This display concept provides a salient representation of two types of boundaries that impact the mission plan: 1) threats to secrecy, and 2) threats to asset preservation. By distinguishing between the two classes of threats, this concept supports human-automation interaction. These two classes of threats may be treated quite differently by a human operator (e.g., a human may initially specify a goal of not crossing either boundary, but as the mission evolves, a "blue" boundary might be crossed much more readily than a "red" boundary if the mission necessitates). An automated planning system, however, may not apply the same level of distinction between these types of threats (e.g., an "intrinsic" optimization goal or "explicit" constraint from the human may cause the automation system to avoid crossing a "blue" threat boundary even though the human operator would not consider it a salient threat to the mission). By providing the operator with salient representations of both types of environmental constraints, this type of display would facilitate humanautomation interaction

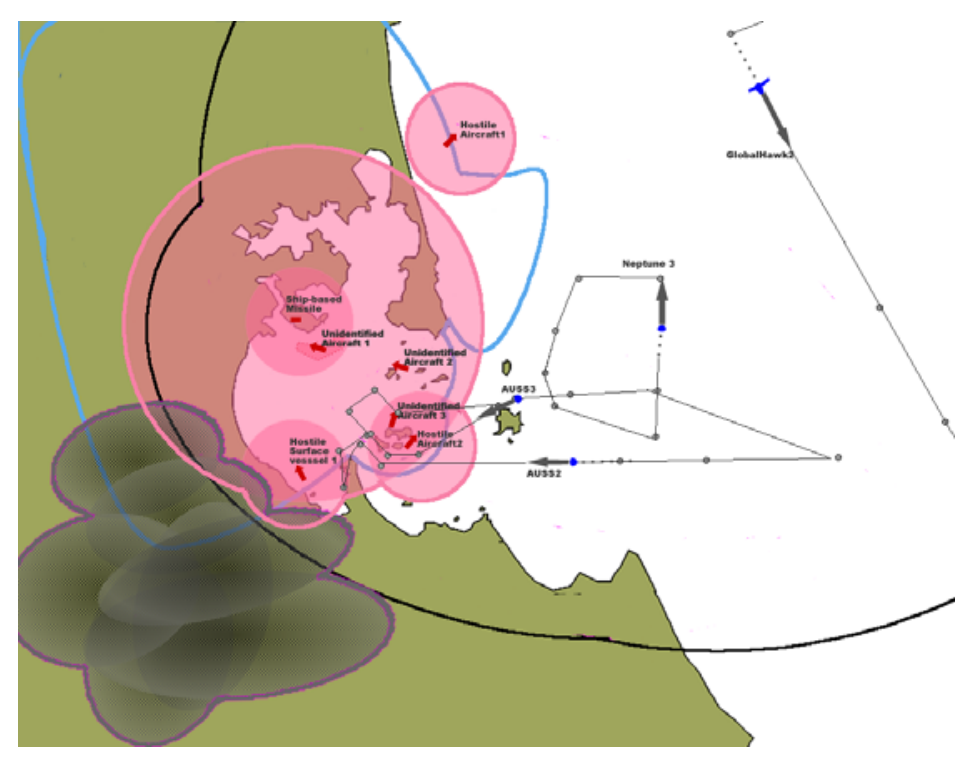

Figure 4. Decomposed “asset preservation” concept 
in defining the evolving level of sensitivity to these different types of threats over the course of the mission.

\section{Decomposition to Support Diagnosis}

Figure 4 shows the same mission environment at the same point in time, but with the display configured to "drill down" into the component parts of the red "asset preservation" boundary. This decomposition concept would provide operators with a method to decompose high-level information constructs into their component parts. A decomposition tool would support operators in diagnosing the details of the factors influencing a mission plan.

In this instance, the red threat boundary is decomposed into its component parts. Similar to the previous example, the human and automation might assign different levels of sensitivity to the different types of threats (either as a result of explicit, implicit, or pre-programmed goals). In this instance, the red "asset preservation boundary" is composed of several enemy threat envelopes in the center of the display, with weather conditions (bottom left) and UV range margins (bottom and top-left) also contributing to the overall constraints on the plan. Further decomposition might allow the operator to assess the actual risk associated with specific enemy threats, and adjust the automation system's sensitivity to those threats.

Overall, these figures demonstrate a subset of MiDAS concepts underlying a conceptual collaboration-space. This collaboration-space, currently in prototype form, will be capable of presenting all categories of information identified in the $\mathrm{AH}$, supporting operators in viewing that information at the level of detail appropriate for the particular demands of the current situation, and facilitating operators in maintaining awareness of subtle features of the environment that may influence automated system functioning.

\section{Evaluation of MiDAS Concepts}

The MiDAS display concepts have been presented to several groups of SMEs to elicit their feedback. The feedback has at times been contradictory (e.g. with the some features receiving substantial criticism from one group of SME's and high praise from another group). SMEs that are familiar with IA program automation systems have been generally positive towards the display concepts, while providing notable critiques of problems with the interface. SMEs that are less familiar with IA program automation systems have noted interface problems identified by the first group, but have also been critical towards some of the underlying concepts upon which the prototype is based (e.g. grouping together information from traditionally different categories). We have attempted to use the model of human-automation interaction described above to provide guidance for interpreting the seemingly irreconcilable feedback from different groups of SMEs. Features of the interface that are likely associated with "pre-programmed" aspects of an automation system would be predicted to receive much harsher critiques by SME's than those features associated with "explicit" human goals. By referring back to this model when interpreting SME feedback, we hope to appropriately address feedback critical to producing a usable human-centered interface while withholding judgment on aspects of the interface designed to exploit an evolution in the human operator role and new automated tools.

\section{CONCLUSION}

Understanding a mission plan requires more than an understanding of the planned actions and the environment within which actions will be executed. It requires a rich understanding of the relationships between planned actions, environmental conditions, and the more abstract goals that the actions are intended to achieve. Traditional "human-centered" displays are tailored towards supporting human understanding of the environment and monitoring of actions, but the linkage between abstract goals and concrete properties is typically achieved through other means (e.g. verbal communication, implicit team awareness developed through training). The introduction of automation systems into the mission planning process requires tools that will allow human operators to communicate with automation systems about the linkage of abstract goals with concrete plans and properties of the environment. The ecological analysis and design concepts described in this paper transform the operator's display into a tool that links abstract goals to concrete properties.

\section{ACKNOWLEDGMENT}

This material is based upon work supported by the Office of Naval Research (ONR) under Contract N00014-05-M-0199. Any opinions, findings and conclusions or recommendations expressed in this material are those of the author(s) and do not necessarily reflect the views of ONR.

\section{REFERENCES}

Billman, L., Cristina, C., Balmer, D., and Warner, N. (2005) Multi-UxV Operator Interface: Lessons Learned and Research Gaps. Presentation given at the August 17, 2005 Intelligent Autonomy Program Review.

Burns, C. M. \& Hajdukiewicz, J. R. (2004). Ecological Interface Design. Boca Raton, FL: CRC Press

Parasuraman, R., Sheridan, T., \& Wickens, C. (2000). A Model for Types and Levels of Human Interaction with Automation. IEEE Transactions on Systems, Man, Cybernetics - Part A: Systems And Humans, 30, pp. 286-297.

Vicente, K. J. (1999). Cognitive Work Analysis: Toward Safe, Productive, and Healthy Computer-based Work. Mahwah, N.J.; London, Lawrence Erlbaum Associates. 